\section{Making Anaglyphs in Photoshop}

\author{
Jerry Sedgewick \\ University of Minnesota \\ info@quickphotoshop.com
}

In order to achieve a three dimensional appearance to a pair of two dimensional images, two off-axis images can be produced and colorized. These can be overlayed slightly apart and then viewed through glasses with two differently colored sides, one color for the left eye and another for the right eye in combinations containing red, green or blue colors. These off-axis and colorized images are referred to as anaglyphs.

Off-axis images can be achieved through the use of a tilting stage on a microscope, by physically changing the position of a camera in relation to a still object, or through changing the axis of an optical stack of sections, such as what is created by confocal/CT scans. Some images lend themselves more to a 3D look both by virtue of inherent three dimensionality limited by the resolution of the imaging system. For example, any number of optical sections can be taken by a standard confocal system of a sample with only 2 or 3 microns of depth, but the resulting anaglyph will look "flat" simply because the sample is flat at confocal resolution. On the other hand, a sample with 20 - 30 microns of depth will appear to have greater depth simply because 3D is inherent to the sample at confocal resolution. The $3 \mathrm{D}$ appearance can be enhanced by spacing the two samples at varying distances, but farther apart requires more effort on relaxing the eyes when viewing.

In Photoshop, two grayscale images at two different axes can be colorized using the method that follows. It works by assigning grayscale images to one of three colors. These colors make up the primary colors for light: red, green and blue (RGB). In Photoshop, the red, green and blue components of color images are referred to as channels. In this method, two grayscale images can be assigned to two out of the three RGB channels. The channels that are selected depend upon the means in which the images will be viewed. For example, red/green glasses use the red and green channels.

Fig. 1: The custom filter in Photoshop: In order to convert from 16-bit to 8-bit in Photoshop CS, type 16 into the centermost position and zeros in other pre-numbered positions.

This method can also be used to colorize grayscale images of fluorescent samples, again assigning appropriate colors to each channel. The resulting colors will, however, contain color values that may not be reproducible in publication, because the primaries of light (RGB) must be reproduced using the primary colors of pigments (cyan, magenta, yellow + black: CMYK). That same phenomena can also occur with anaglyphs. If reproducible colors are desired for fluorescent or darkfield images, then it is best to use values suggested in an earlier publication of Microscopy Today (Vol 12/6, November 2004). To make reproducible colors after creating an anaglyph, use methods described on the www. quickphotoshop.com site: click on "RGB to CMYK" link.
Making Anaglyphs in Photoshop. Open the two images that are to be colorized and overlaid. Be sure that these are both grayscale images. If not, change the mode (Under Image $>$ Mode $>$ Grayscale). If the image is 16-bit (versus 8-bit), then adjust brightness by moving the white triangle on the Levels slider to the right end of the histogram while being careful to measure the whitest values in your image so that pixel intensities do not exceed 254 (saturate) in versions of Photoshop previous to CS or Photoshop 8. In Photoshop $\mathrm{CS}$, under Filter $>$ Other $>$ Custom, type 16 in the center of the grid and eliminate all other values (see fig. 1). Then change the mode (Under Image $>$ Mode $>8$-bits/Channel).

In order to merge three channels when only two images are opened, a third "dummy" channel needs to be created. The image that will become the third channel must be at the same resolution (pixels across by pixels down) as the other two. That is easily done by duplicating one of the two opened images (Under Image $>$ Duplicate). This image needs to be completely black in order to contain no values to be colorized. Select all of the duplicated image (under Select $>$ Select All, or Control/Command + A key), then fill with black (Under Edit $>$ Fill: in the Fill dialogue box, click on the drop down arrowhead adjacent to Use and select Black).

Now all three images can be overlaid into the same image. That can be accomplished by opening the Channels dialogue box (under Window $>$ Channels or Show Channels). Click on the arrowhead in the top, right corner to reveal a drop down list. From that list, choose Merge Channels. A dialogue box will open to prompt you for the mode of the image and the number of channels. Choose RGB for the mode, and 3 for the number of channels. Another dialogue box will open to prompt you for the image used for each channel. Assign each of the three opened images to their respective channels.

Now the image can be viewed through glasses. Alterations to tilt and spacing may have to be made to improve the 3D look. Alterations to the tilt cannot be done in Photoshop, but spacing can be altered. To do that, the RGB image can be split apart again and the channel(s) of interest can be shifted laterally. Split the image into separate channels

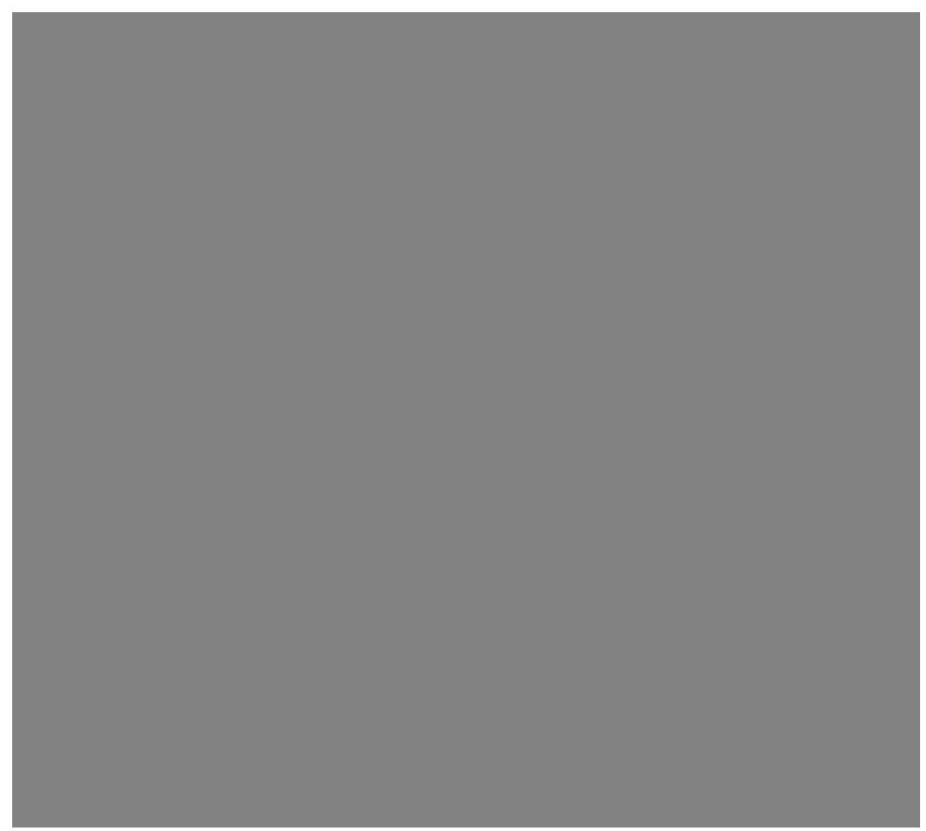

Fig 2: Anaglyph of a fly taken on a macroscope with a eucentric stage. The lateral positioning of the two, overlaid fly images are far apart to emphasize three-dimensionality. This image is viewed with red/blue glasses. 


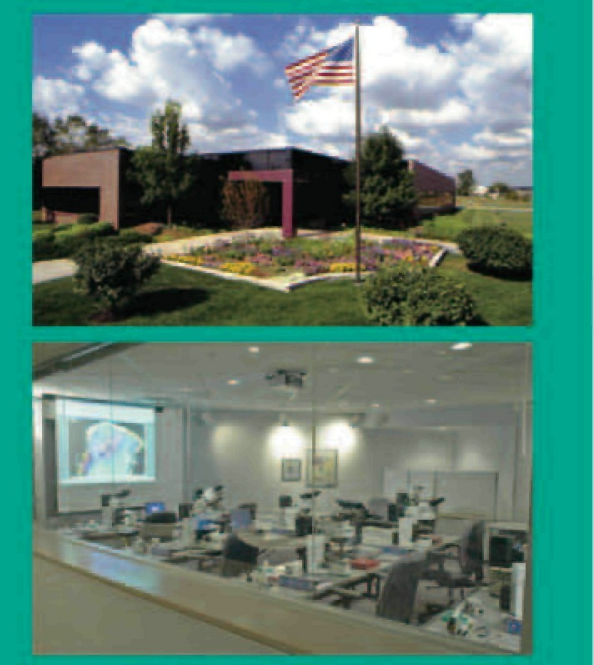

of COLLEGE OF MICROSCOPY

Located in Westmont, Illinois, the College of Microscopy is an institution for specialized instruction and education, whose goal is to advance the knowledge and understanding of light and electron microscopy for materials analysis.

One of the advantages of attending a course at the College of Microscopy is that our courses are taught primarily by experienced senior staff scientists from McCrone Associates, Inc. who solve micro-analytical problems on a daily basis. That's why it's true when we say,

\section{"Learn From Experience... \\ Learn From The Experts."}

Microscopical Identification of White-Powder Unknowns (Part 1) June 6-10, 2005

Microscopic Particle Handling:

Particle Isolation, Manipulation, and Mounting

October 3-7, 2005

Microscopical Identification of Pharmaceutical Materials
and Contaminants
July $11-15,2005$

Microscopical Particle Analysis

August 8-12, 2005

Microscopical Examination of Forensic

Trace Evidence Particles (Part 1)

September 12-16, 2005

Microscopical Identification of Pigments for Art Conservation and Architectural Restoration Professionals

September 19-23, 2005

\section{Scanning Electron Microscopy}

October 17-21, 2005

Polarized Light and Chemical Microscopy

November 14-18, 2005

630-887-7100

www.collegeofmicroscopy.com 


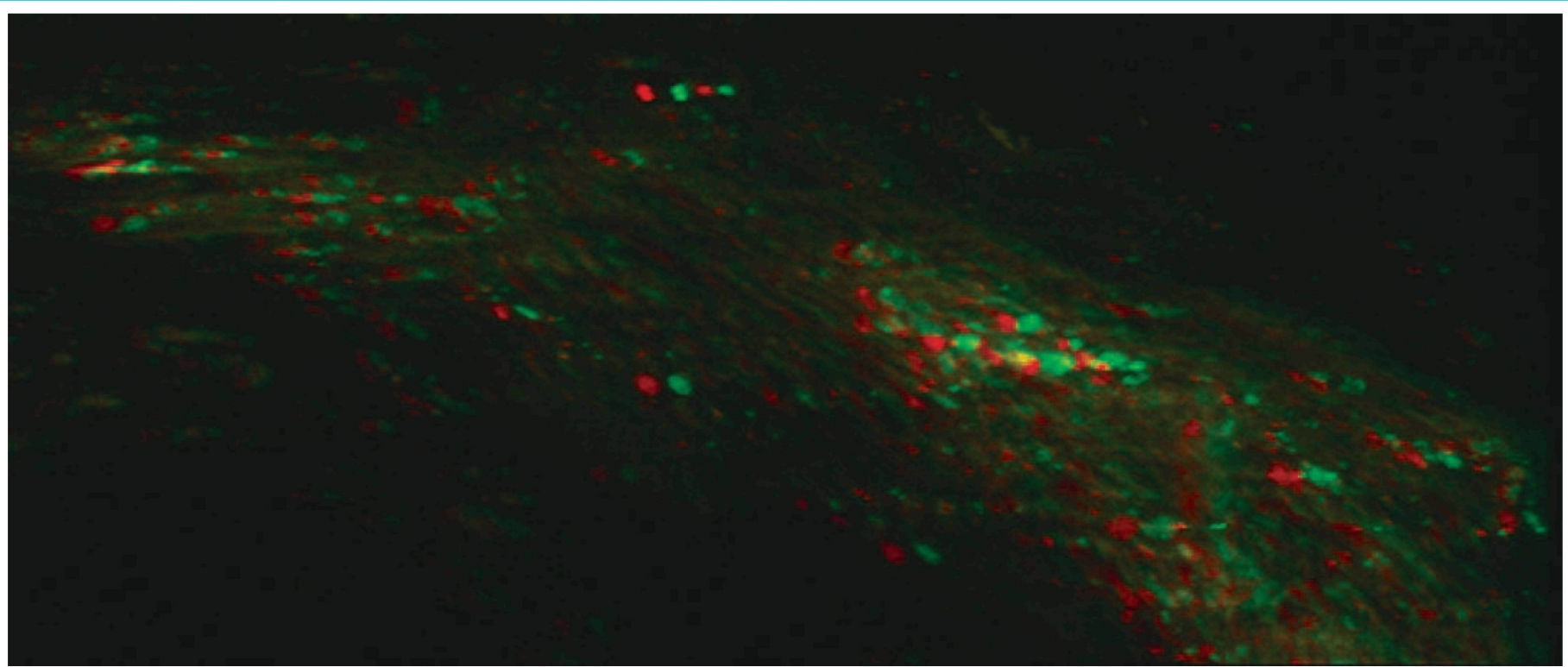

Fig 3: Confocal optical sections through a 40 micron sample at 4 micron $z$-steps in two colors, red and green. This sample lends itself to a three dimensional look.

again by clicking on the arrowhead to reveal the drop down list, and then choose Split Channels.

On the image of interest, move the image laterally into the desired position. That position may have to be iteratively found by repositioning laterally, merging channels to view, and then repeating the process until the best $3 \mathrm{D}$ positioning is found.

Before the image can be moved, the locked background layer must be duplicated and the locked layer deleted. Adobe instituted the locked background layer as a default functionality built into Photoshop in versions later than $5 \mathrm{x}$ so that original images aren't accidentally altered, but the result is that many procedures cannot be accomplished unless the locked layer is "unlocked." To do that, find the Layers dialogue box or open the Layers box under Window>Layers or Show Layers. In the Layers dialogue box, click on the arrowhead in the upper, right to reveal a drop down list. From the list choose Duplicate Layer. You will then need to eliminate the background layer, done by clicking on the background layer to highlight it, then using the drop down list to select Delete Layer.

On the image(s) of interest, click on the move tool from the toolbar. While holding down the Shift key in order to limit the movement only to the horizontal axis, move the image by clicking and dragging with the mouse. The image can also be moved with the arrow keys in single pixel movements, or by holding the shift key down while using the arrow keys in order to move the image in 10 pixel increments.

Images can again be merged by selecting Merge Channels from the drop down list in the Channels dialogue box. Crop out any extra border that was created at the edges if you moved the images laterally (Use the crop tool from toolbar and double click inside area when satisfied with outlining area of interest: boxes at corners can be used to expand and contract the size of the outline by clicking inside the box and dragging to desired position).

Optical z-stacks can also be made manually into anaglyphs, though programs like Confocal Assistant and often software included with confocal instruments that can make these with a click of a button. To accomplish this manually with one fluorophore represented only, make a second copy of a confocal stack in operating system software using a different name. Then rotate one of the two optical stacks anywhere from 6 - 10 degrees in the $\mathrm{x}$ or $\mathrm{y}$ position, depending as you desire. Use the method outlined above to colorize and merge into appropriate two colors, depending upon the glasses that are used to obtain a 3D view.

These images lend themselves far more to audience interaction, and provide information about the nature of the images in three dimensions.

Special thanks to Ted Clarke for the fly anaglyph image.

Light Guides \&
Custom Scintillators
from
M. E. Tay/or Engineering, Inc
Light Guides for Leo/Cambridge model SEMS
from \$495
ITO Gold and Custom Scintillators from $\$ 89$
P47 Phospor, YAG, YAP and many others
Highest Quality Engineering
See our web site for special offers on
Maintenance and recoating specials
E. Taylor Engineering, Inc.
21604 Gentry Lane
Brookeville, MD 20833
Phone: (301) 774-6246
Visit us on the web:
www.semsupplies.com




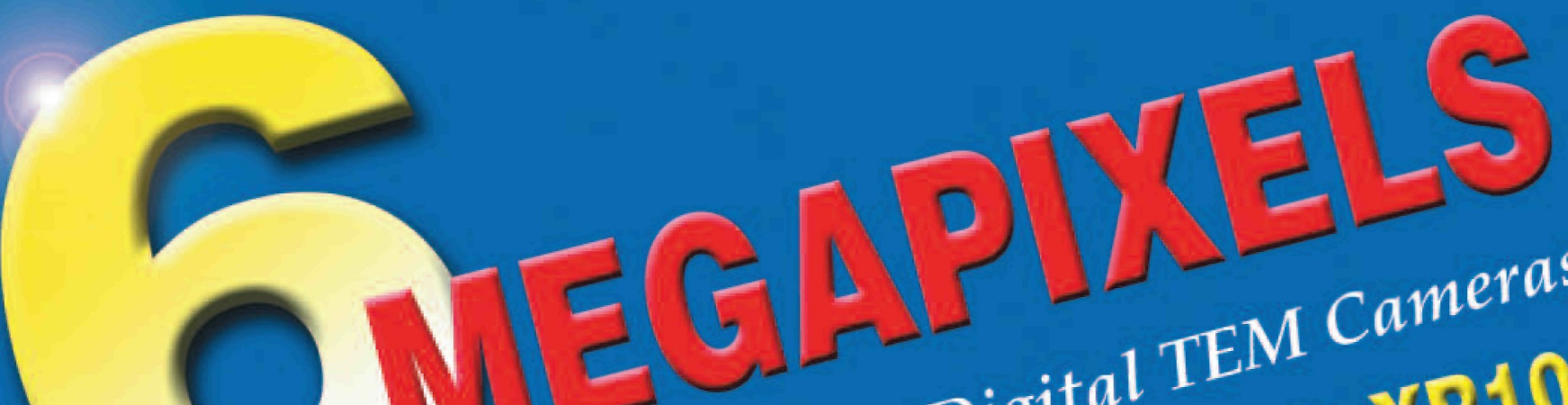
High Definition Digi

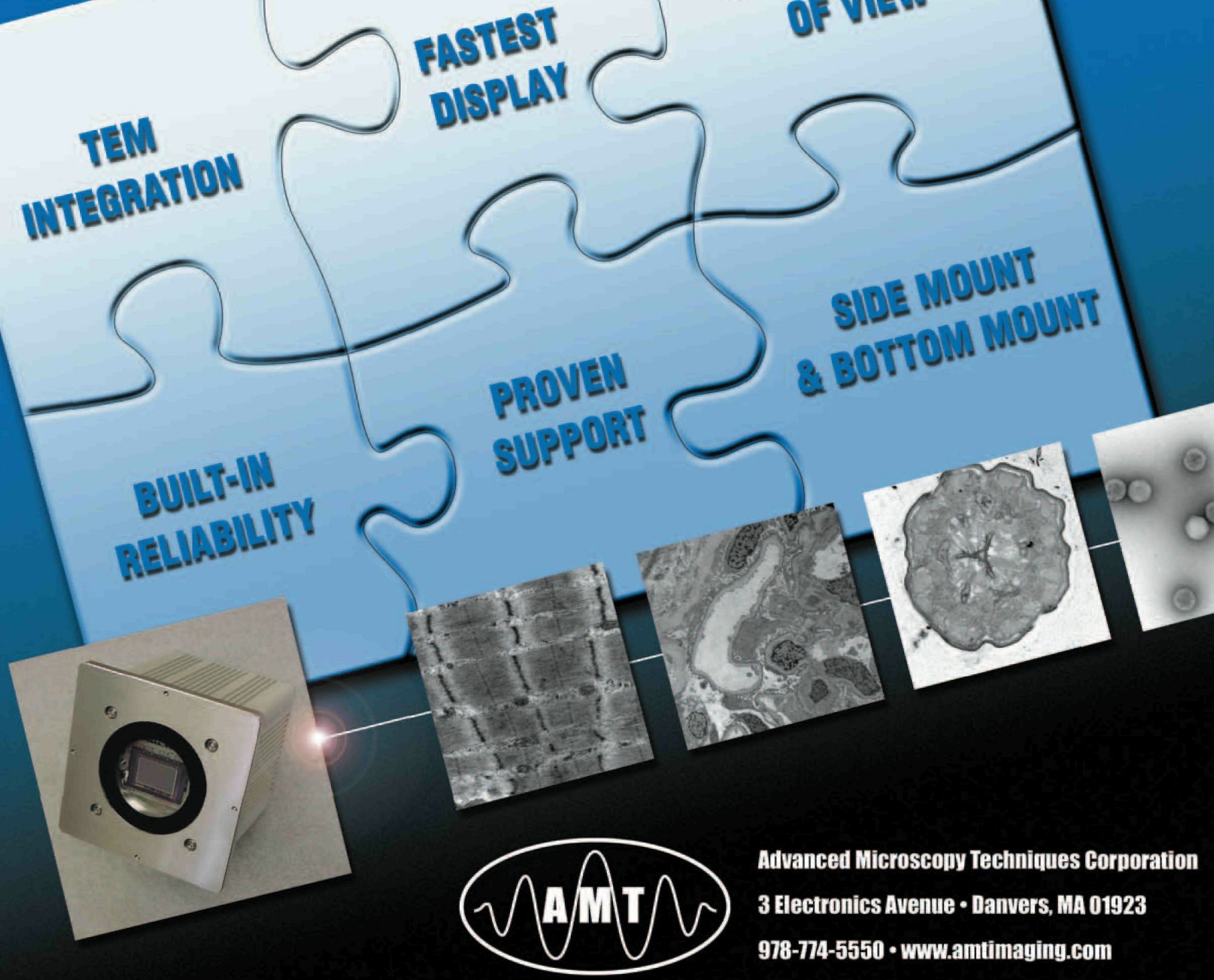

